\title{
Tissue expression of Squamous Cellular Carcinoma Antigen (SCCA) is inversely correlated to tumor size in HCC
}

\author{
Paolo Trerotoli $^{\dagger 1}$, Emilia Fransvea ${ }^{\dagger 2}$, Umberto Angelotti ${ }^{2}$, \\ Giovanni Antonaci ${ }^{2}$, Luigi Lupo ${ }^{3}$, Antonio Mazzocca ${ }^{2,4}$, Anita Mangia ${ }^{5}$, \\ Salvatore Antonaci ${ }^{2}$ and Gianluigi Giannelli*2
}

\begin{abstract}
Address: ${ }^{1}$ Department of Biomedical Science and Human Oncology, Section of Medical Statistics, University of Bari Medical School, Bari, Italy, ${ }^{2}$ Department of Internal Medicine, Immunology and Infectious Diseases, Section of Internal Medicine, University of Bari Medical School, Bari, Italy, ${ }^{3}$ Department of Emergency and Organ Transplantation, University of Bari Medical School, Bari, Italy, ${ }^{4}$ Vanderbilt University Medical Center Department of Pathology, Nashville, USA and ${ }^{5}$ Clinical Experimental Oncology Laboratory, National Cancer Institute Bari, Italy

Email: Paolo Trerotoli - ptrerotoli@stamed.uniba.it; Emilia Fransvea - emifra@yahoo.com; Umberto Angelotti - angelo@yahoo.com; Giovanni Antonaci - ganto@yahoo.com; Luigi Lupo - lupo@clichiru.uniba.it; Antonio Mazzocca - antonio.mazzocca@vanderbilt.edu; Anita Mangia - anita.mangia@irccs.org; Salvatore Antonaci - s.antonaci@intmed.uniba.it; Gianluigi Giannelli* - g.giannelli@intmed.uniba.it

* Corresponding author †Equal contributors
\end{abstract}

Published: 27 May 2009

Molecular Cancer 2009, 8:29 doi:10.1 186/1476-4598-8-29
Received: 23 January 2009

Accepted: 27 May 2009

This article is available from: http://www.molecular-cancer.com/content/8/1/29

(C) 2009 Trerotoli et al; licensee BioMed Central Ltd.

This is an Open Access article distributed under the terms of the Creative Commons Attribution License (http://creativecommons.org/licenses/by/2.0), which permits unrestricted use, distribution, and reproduction in any medium, provided the original work is properly cited.

\begin{abstract}
Background: This study aimed to investigate squamous cellular carcinoma antigen (SCCA) in serum and in tumoral and paired peritumoral tissues. We studied 27 patients with liver cirrhosis (LC) and 55 with HCC: 20 with a single nodule $<3 \mathrm{~cm}(\mathrm{~s}-\mathrm{HCC})$ and 35 with a single nodule $>3$ $\mathrm{cm}$ or multifocal (I-HCC).

Methods: Serum SCCA was measured by the ELISA kit, and in frozen tissues by immunohistochemistry, quantified with appropriate imaging analysis software and expressed in square microns. Continuous variables are reported as means and $95 \%$ confidence intervals. Comparisons between independent groups were performed with a generalized linear model and Tukey grouping. Pearson's correlation coefficients were determined to evaluate relations between markers. Qualitative variables were summarized as count and percentage. Statistical significance was set at $p$-value $<0.05$.

Results: Serum SCCA values in LC patients were $0.4 \mathrm{I}(0.3 \mathrm{I}-0.55) \mathrm{ng} / \mathrm{ml}$ and statistically different from both HCC groups: 1.6 (I.0-2.6) ng/ml in s-HCC, 2.2 (I.28-2.74) ng/ml in I-HCC. SCCA in hepatic tissue was 263.8 (I 76.6-394.0I) $\mu \mathrm{m}^{2}$ in LC patients, statistically different from values in sHCC: II63.2 (863.6-I566.8) $\mu^{2}$ and I-HCC: 625.8 (534.5-732.6). All pairwise comparisons between groups yielded statistically significant differences. Tumoral SCCA resulted linearly related with nodule size, showing a statistically significant inverse relation between the two variables $(b=$ $-0.099, p=0.024)$.

Conclusion: There was no statistically significant correlation between tissue and serum levels of SCCA. The significantly stronger expression of SCCA in smaller compared to larger HCC could be important for early HCC detection. However, the increased expression in peritumoral tissue could affect the significance of serological detection.
\end{abstract}




\section{Background}

Early recognition of the onset of hepatocellular carcinoma (HCC) would help to select more effective therapies for patients, leading to a better prognosis and life span. For this reason surveillance programs were strongly recommended at a Consensus Conference held in Barcelona [1]. Alphafetoprotein (AFP), the only marker commonly used in clinical practise, displays poor sensitivity and a high specificity only for values higher than $400 \mathrm{IU} / \mathrm{ml}$. However, because AFP concentrations are directly correlated with tumor size, the reliability of such a marker appears inadequate for early recognition of HCC [2]. This has prompted a high number of studies conducted to validate different new biomarkers, but very little has yet been reported about biomarkers helping to achieve an early detection of HCC [3]. All the proposed biomarkers failed to discriminate between liver cirrhosis (LC) and HCC in a satisfactory manner, in terms of diagnostic accuracy, reproducibility of the results, or technical issues related to the biomarker detection method [4]. For this reason, the simultaneous use of different tests seems to offer a promising approach that warrants further investigation [5].

Squamous cellular carcinoma antigen (SCCA), is a member of the high molecular weight family of serine protease inhibitors named serpins [6]. Two highly homologous isoforms have been reported to be expressed in HCC tissues at protein and translational levels [7]. SCCA has also been reported to be overexpressed in tumoral compared to paired peritumoral tissue of HCC, suggesting a role as a potential marker for histological detection of HCC [8]. Recently, SCCA has been investigated in regenerative and dysplastic nodules of HCC tissue. Interestingly, results show that SCCA was poorly expressed in regenerative tissue but strongly increased in dysplastic nodules, suggesting a role as a potential marker for early detection of HCC [9].

Aim of this study is to investigate the tissue expression of SCCA in patients with different characteristics of HCC, namely small and large or multifocal nodules.

\section{Results}

SCCA was quantified in matching sera and tissues of 82 patients, 27 LC and 55 HCC. In these latter patients tissue expression of SCCA was investigated in neoplastic and paired peritumoral tissue. Patients were stratified according to nodule size. The mean $(95 \% \mathrm{CI})$ size of the HCC lesion was $4.07 \pm 2.08 \mathrm{~cm} ; 36.4 \%(20 / 55)$ patients had a single nodule smaller or equal to $3 \mathrm{~cm}$ (s-HCC) and $63.6 \%(35 / 55)$ a nodule larger than $3 \mathrm{~cm}$ or multifocal (lHCC). A statistically significant difference $(p=0.01)$ was observed in the percentage of Child-Pugh stage A among s-HCC (55\%), l-HCC (82\%) and LC (88\%). The most frequent etiology was HCV alone or with HBV, with no significant difference among groups $(\mathrm{p}=0.108): 65 \%(15 /$ $20)$ in s-HCC, $71.4 \%(25 / 35)$ l-HCC and $92.6 \%(25 / 27)$ for LC patients (Table 1).

Table I: Characteristics of the patients

\begin{tabular}{|c|c|c|c|c|c|}
\hline & & Single nodule $\leq 3 \mathrm{~cm}$ & Single nodule $>3 \mathrm{~cm}$ & Total HCC & LC \\
\hline \multirow[t]{2}{*}{ Sex } & $M$ & 16 & 28 & 44 & 17 \\
\hline & $\mathrm{F}$ & 4 & 7 & 11 & 10 \\
\hline Age & & $65(10.15)$ & $65(10.2)$ & $65(10.1)$ & \\
\hline Size & & $2 . .4(0.5)$ & $5(2)$ & $4(2.1)$ & \\
\hline \multirow[t]{4}{*}{ CLIP } & 0 & 7 & 15 & 22 & \\
\hline & I & 10 & 17 & 27 & \\
\hline & 2 & 3 & 3 & 6 & \\
\hline & & & & & 27 \\
\hline \multirow[t]{2}{*}{ CHILD } & $A$ & 11 & 29 & 40 & 24 \\
\hline & B & 9 & 6 & 15 & 3 \\
\hline \multirow[t]{8}{*}{ Etiology } & Alcohol or Other & 1 & 2 & 3 & I \\
\hline & HBV & 3 & 8 & 11 & I \\
\hline & $\mathrm{HBV}+\mathrm{Alcohol}$ & 1 & & I & \\
\hline & Total HBV & 4 & 8 & 12 & I \\
\hline & $\mathrm{HCV}$ alone & 13 & 23 & 36 & 24 \\
\hline & $\mathrm{HCV}+\mathrm{HBV}$ & 1 & 2 & 3 & \\
\hline & $\mathrm{HCV}+\mathrm{Alcohol}$ or other & I & & I & I \\
\hline & Total HCV & 15 & 25 & 40 & 25 \\
\hline
\end{tabular}


SCCA was detected by immunohistochemistry in all the patients, although with some differences, in both tumoral and peritumoral tissues of small and large HCC (Figure 1A). In particular, the mean (95\% CI) expression in neoplastic tissue was $1163.2(863.6-1566.8) \mu \mathrm{m}^{2}$ in s-HCC and $625.8(534.5-732.6) \mu^{2}$ in l-HCC. There was a statistically significant difference among the groups $(\mathrm{F}=$ $17.45, \mathrm{p}=0.002$ ) and Tukey grouping showed a significant difference $(\mathrm{p}<0.05)$ between s-HCC vs l-HCC (Figure $1 \mathrm{~B}$ ). To further investigate the tissue expression of SCCA, paired peritumoral tissues were analyzed as well as LC samples used as proper control. The mean (95\% CI) of SCCA tissue expression was $263.8(176.6-394.01) \mu \mathrm{m}^{2}$ in s-HCC peritumoral tissue, $345.49(263,98-452,16) \mu \mathrm{m}^{2}$ in l-HCC and $232.63(163.3-331.38) \mu^{2}$ in LC, Figure 2. The model did not result statistically significant $(\mathrm{F}=1.84$, $\mathrm{p}=0.16)$. In conclusion, SCCA was more strongly expressed in the neoplastic tissue of smaller compared to larger HCC. In addition, it is noteworthy that the ratio between tumoral and peritumoral SCCA shows a trend ranging from 4.4 in s-HCC to 1.8 of l-HCC.

In the same patients, we measured the serum concentrations of SCCA. As reported in Figure 3, concentrations were $1.6(1.02-2.6) \mathrm{ng} / \mathrm{ml}$ in s-HCC, $2.2(1.28-2.74) \mathrm{ng} /$ $\mathrm{ml}$ in l-HCC, $0.41(0.31-0.55) \mathrm{ng} / \mathrm{ml}$ in LC patients (Figure 3$)$. The model resulted statistically significant $(\mathrm{F}=$ $20.81, \mathrm{p}<0.0001$ ), and Tukey grouping displayed a significant difference $(\mathrm{p}<0.05)$ between each HCC group and LC. In conclusion, serum SCCA levels were similar in the different HCC groups, but statistically different in the LC group.

The underlying liver disease does not seem to affect SCCA tissue expression levels among patients with different Child-Pugh stages $(\mathrm{p}=0.5)$ as shown by the generalized linear model, while the only significant factor remains the diagnosis of HCC as compared to LC ( $p=0.049)$.

The regression model with dependent peritumoral SCCA shows that in the subgroup of single nodule HCC, nodule size was not statistically significant as a means of predicting peritumoral SCCA $(b=0.064 ; \mathrm{F}=1.55 ; \mathrm{p}=0.219)$, whereas it was a statistically significant predictor of tumoral SCCA $\left(\mathrm{b}=-0.099 ; \mathrm{F}=5.47 ; \mathrm{p}=0.024 ; \mathrm{R}^{2}=\right.$ $0.117)$.

Linear regression between serum SCCA and nodule size did not show a statistically significant relation between these two variables $\left(\mathrm{b}=0.023 ; \mathrm{F}=0.14 ; \mathrm{p}=0.71 ; \mathrm{R}^{2}=\right.$ $0.003)$.

In conclusion, tumoral SCCA depends on nodule size, and there was an inverse trend between nodule size and higher SCCA values. Peritumoral and serum SCCA, instead, do not show any relation with tumor size.

The area under the ROC curve for serum SCCA was 0.897 (CI95\% 0.81-0.953), with a suggested cut-off value of 1.1 $\mathrm{ng} / \mathrm{ml}$, showing $72.7 \%$ sensitivity, $100 \%$ specificity and a 72.7\%Youden index. Assessing only s-HCC and LC patients, an analogous accuracy of serum SCCA was obtained: AUC 0.873 (CI95\% 0.743-0.952), cut-off 1.1 $\mathrm{ng} / \mathrm{ml}$, with $70 \%$ sensitivity, $100 \%$ specificity and $70 \%$ Youden index.

However, in HCC no statistically significant correlation was observed between SCCA levels present in the tumoral and/or peritumoral tissue and in the serum (Table 2). Pearson's correlation coefficient resulted statistically significant only to evaluate relations between serum levels vs cirrhotic liver tissue expression of SCCA in LC patients: 0.39 ( $\mathrm{p}=0.04)$. In other words, the higher the tissue values the higher the serum values, but only in LC patients.

Finally, serum SCCA values could be expressed as a function of tumoral or peritumoral values of this marker, so a regression analysis was performed; results are shown in Figure 4 . The models evaluated did not result statistically significant except in LC patients $(\mathrm{F}=5.02, \mathrm{p}=0.034)$. In this group the slope resulted $0.33(\mathrm{p}=0.034)$, suggesting increased SCCA serum values with stronger tissue expression. In HCC patients, neither SCCA tumoral nor peritumoral tissue expression resulted predictive of the serum levels, and this finding was consistent in both groups of patients. Furthermore, in smaller nodules we calculated that $711 \mu \mathrm{m}^{2}$ of SCCA antigen were necessary to measure one unit in the serum, while in larger tumors only 268 $\mu \mathrm{m}^{2}$ were enough.

\section{Discussion}

Lately, the investigation of new biomarkers for HCC diagnosis has aroused great interest because they could make it possible to select the most effective therapy for individual patients. From this viewpoint, biomarkers helping to detect small HCC would be a great step forward. Recently, the tissue expression of SCCA has been reported in a higher percentage of patients with pre-neoplastic dysplastic lesions than in regenerative nodules. Moreover, in the same study SCCA was reported to be more strongly expressed in premalignant dysplastic nodules than in HCC [9]. In our study a similar statistically significant difference was observed, but because of the small number of patients ( 5 LC vs. 20 s-HCC patients) with dysplastic cirrhotic nodules, no reliable conclusion can be drawn although it seems an interesting initial observation. Consistently with these data, we report herein that SCCA is more strongly expressed in the tissue of smaller as com- 
A
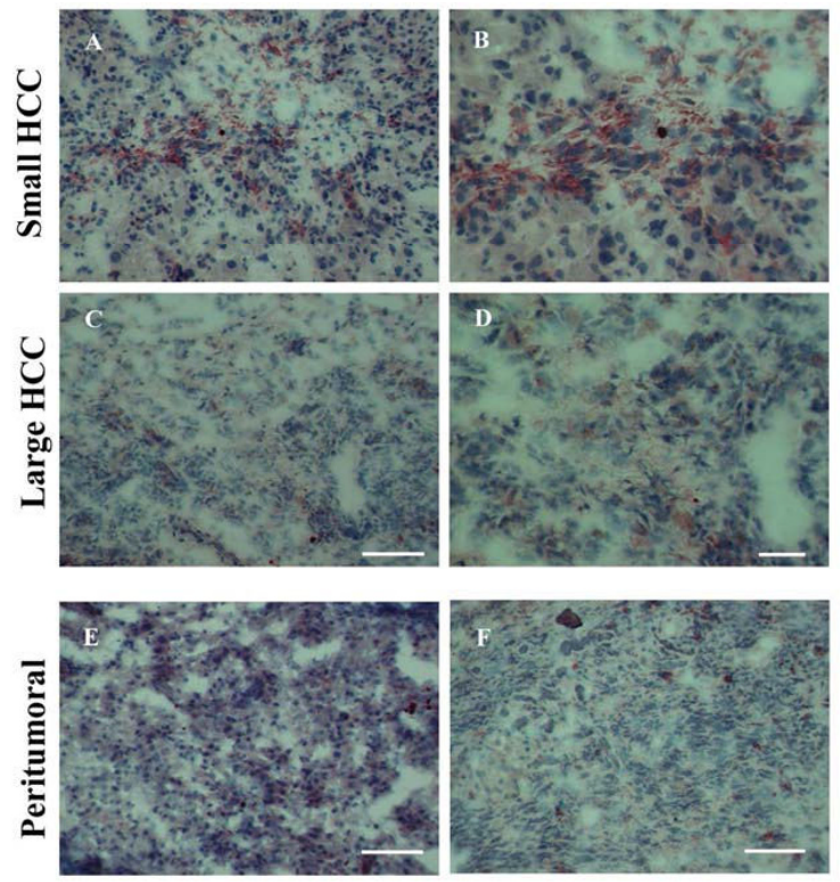

B

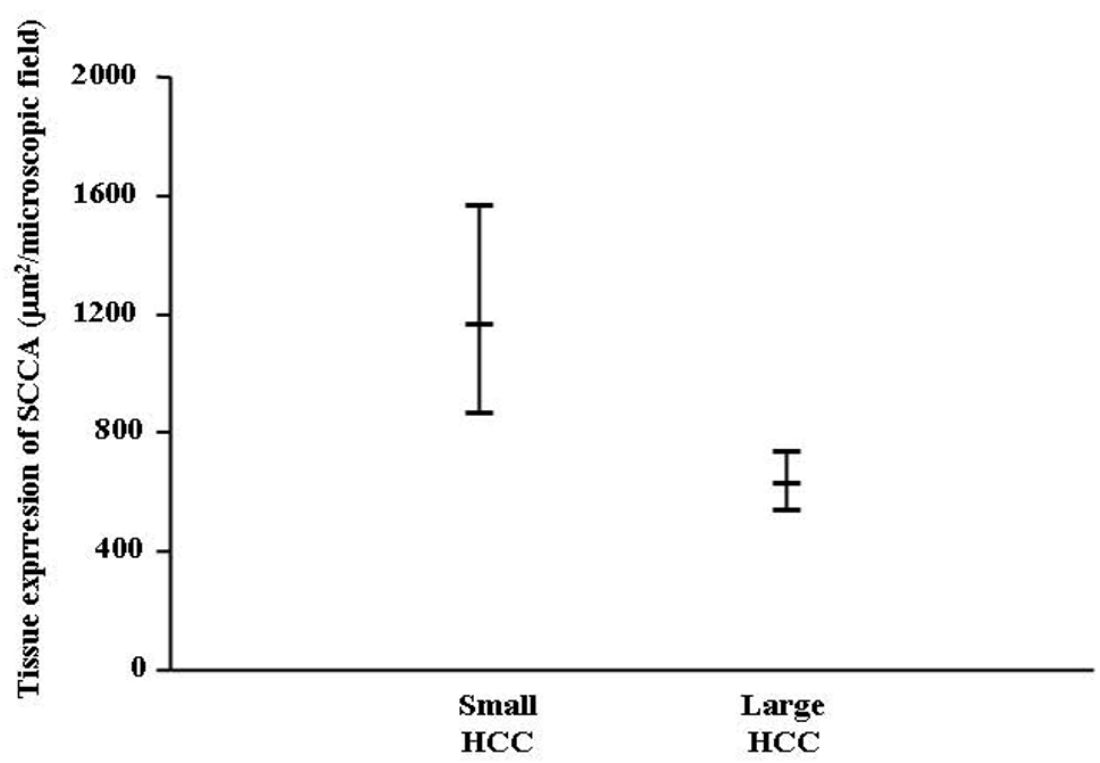

Figure I

Tissue expression of SCCA. In Figure IA, immunohistochemistry of SCCA in small and large HCC at low (A, C) and high $(B, D)$ magnification. Paired peritumoral tissue at low magnification of small $(E)$ and large $(F) H C C$. In $A, C, E$ and $F$ scale bar $=$ $100 \mu \mathrm{m}$, in B and D scale bar $=50 \mu \mathrm{m}$. In Figure IB, 95\% Confidence intervals of neoplastic tissue SCCA in the different HCC patients groups. 


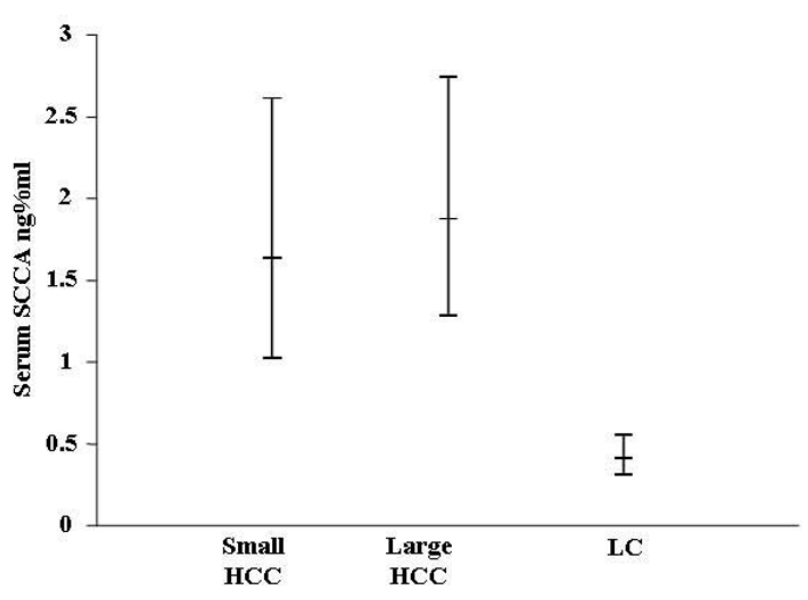

Figure 2

95\% Confidence intervals of non neoplastic peritumoral tissue SCCA in the different HCC patients groups.

pared to larger HCC. For the first time, therefore, a marker that can discriminate smaller better than larger nodules is reported, although findings of this marker also in the tissue limit its immediate application to clinical practise. Nevertheless, results in this study contain two different messages, firstly that SCCA could be a biomarker of premalignant transformation and secondly, that the irregular behavior of this serum biomarker could be related to a different biological tumor status.

In particular, the decreased SCCA expression with the progression of tumor size, and the increased expression in the peritumoral tissue of larger HCC at higher risk of further neoplastic transformation support our first conclusion.

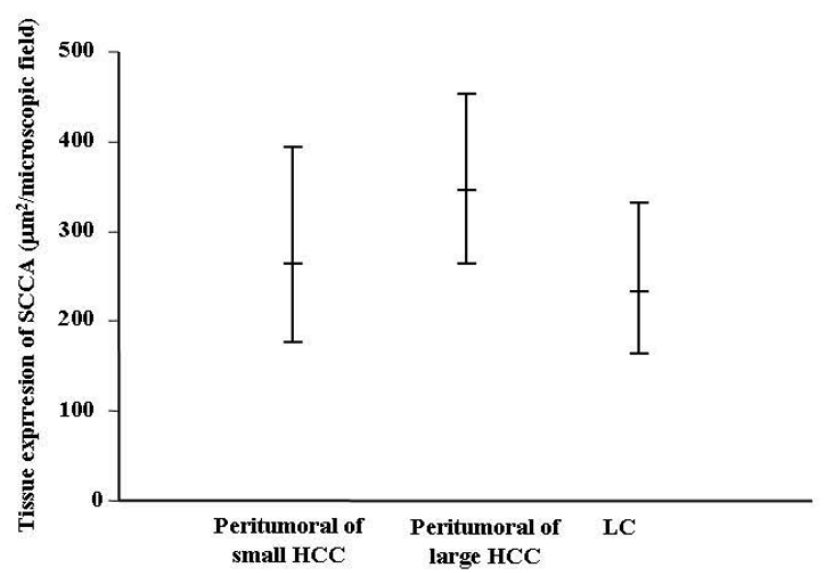

Figure 3

95\% Confidence intervals of serum SCCA in the different HCC patients groups.
Table 2: Pearson correlation coefficient for evaluation of relation between markers in HCC and LC patients

\begin{tabular}{cccc}
\hline & & Peritumoral SCCA & Tumoral SCCA \\
\hline s-HCC & Serum SCCA & 0.01 & -0.08 \\
\hline & Tumoral SCCA & 0.27 & \\
\hline & & & -0.13 \\
\hline I-HCC & Serum SCCA & 0.05 & \\
\hline & Tumoral SCCA & 0.014 & \\
\hline & & \\
\hline LC & Serum SCCA & $0.39(\mathrm{P}=0.04)$ \\
\hline
\end{tabular}

These results are consistent with our previous observation [5], and are also supported by a previous report by Pontisso et al. showing that in patients with LC progressing to HCC, SCCA was consistently increased [10]. This could explain why SCCA could be unexpectedly increased in some LC patients. In fact, the relation between nodule size and tumoral SCCA in our study suggests that high levels of SCCA expression could anticipate clinical evidence of HCC onset.

On the other hand, the lack of correlation between tissue and serum SCCA levels is disappointing and no explanation is yet forthcoming. However, the fact that SCCA is distributed mainly in the cytosol, not associated to membrane-bound vesicles, and therefore is not properly secreted but rather released in the serum as a consequence of cell lysis, could contribute to clarify this issue [11]. This hypothesis is also confirmed by our previous study showing SCCA expression in some cell lines but not in the conditioned medium, suggesting a defective protein secretion [8].

Moreover, it is noteworthy that the amount of serum SCCA does not depend on its expression level in the peritumoral or LC tissue. The higher ratio in smaller than larger nodules suggests that SCCA is produced and released at different times, likely during the earlier events in HCC progression; this offers another possible explanation of the discrepancy between SCCA serum and tissue levels.

In conclusion, this study suggests that SCCA tissue expression could be a marker for early detection of smaller HCC nodules, and contributes to explain why the data in serum can be somewhat disappointing. In addition, our results suggest that proposed biomarkers need to be carefully investigated and clinically validated in relation to specific 
LC

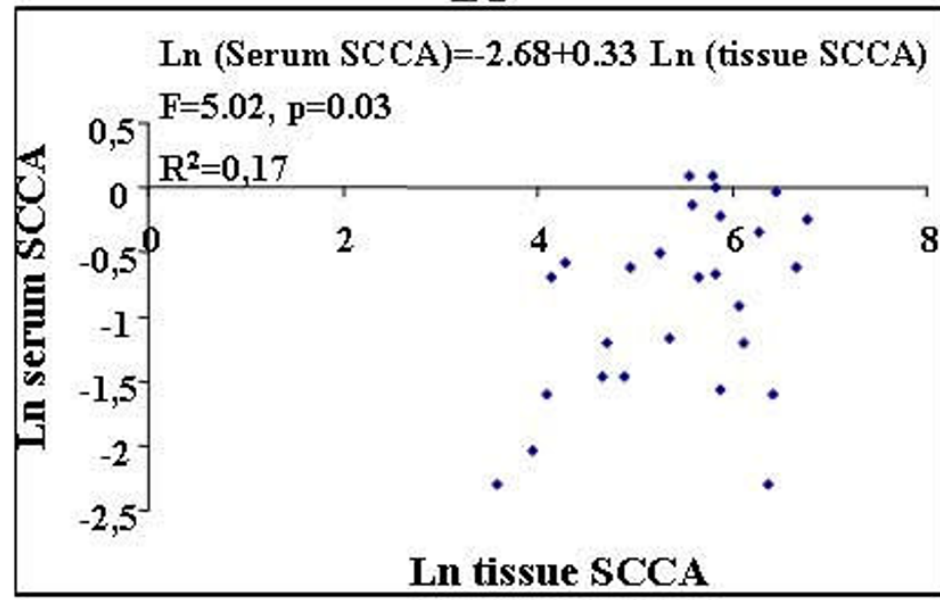

HCC

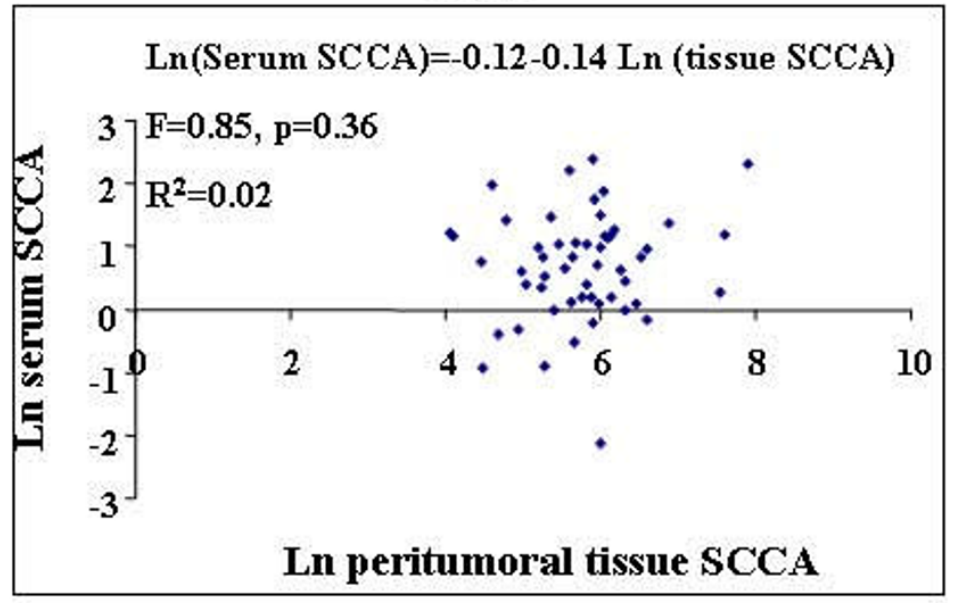

\section{HCC}

$\operatorname{Ln}($ Serum $\mathrm{SCCA})=2.55-0.28 \mathrm{Ln}$ (tissue $\mathrm{SCCA})$

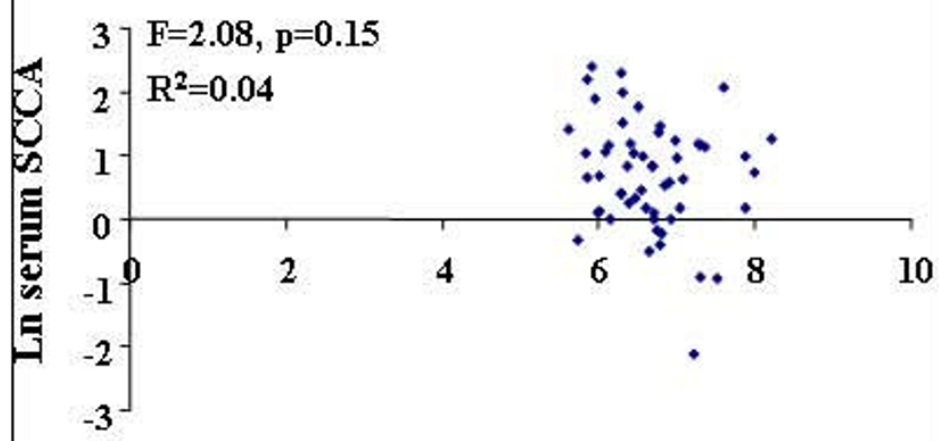

Ln tumoral tissue SCCA

Figure 4

Scatter plot of serum SCCA in tumoral and peritumoral tissue in HCC patients, and tissue SCCA in LC patients. $\mathrm{Ln}=$ natural logarithm. 
biological aspects of HCC, so as to obtain more reliable results.

\section{Materials and methods \\ Tissue and serum collection}

Tissue specimens of primary nodules and of the peritumoral area were collected from HCC patients, as well as specimens from LC patients. All the specimens, obtained by surgical biopsy, were fixed in 3.7\% formaldehyde and processed for routine histology, while a part of the specimen was immediately snap-frozen in liquid nitrogen and stored at $-80^{\circ} \mathrm{C}$ until use. Serum samples from the same patients were collected before any kind of treatment, and stored at $-20^{\circ} \mathrm{C}$ until use.

Patients were classified as LC and HCC according to EASL criteria, and tumor staging was determined according to the CLIP classification [1,12]; nodule size was determined by concordant US and CT and/or MRI scans. SCCA was quantified in matching sera and tissues of 82 patients, 27 LC and 55 HCC. In the latter patients, tissue expression of SCCA was investigated in both neoplastic and paired peritumoral tissue.

The study was performed in accordance with the Helsinki declaration and informed written consent was obtained from all patients before surgery or liver biopsy and before blood sample collection

\section{Tissue expression of SCCA}

Immunohistochemistry was performed on frozen specimens as previously reported [13]. SCCA was detected using a polyclonal antibody directed against recombinant SCCA purchased from (Hepa-Ab, Xeptagen, Italy) and following the manufacturer's instructions.

The expression of SCCA was measured as $\mu \mathrm{m}^{2}$ of staining by an appropriate software system (Lucia, Nikon, Corp), already validated in several of our previous reports. Briefly, expression of the SCCA antigen was measured in each section as the total stained area, calculated as the mean of ten randomly chosen microscopic fields. Figures 1 and 2 show the mean and standard deviation among all those calculated. To normalize the quantification of the staining, a negative control (a section incubated without the primary antibody) was included in each experiment, so that the sensitivity of the software was calibrated on the background staining.

\section{Serum determination of SCCA}

Serum determination of the SCCA antigen was carried out using an ELISA kit purchased from Xeptagen (Xeptagen, Naples, Italy) following the manufacturer's instructions as previously reported [8]. Briefly, the SCCA ELISA kit is based on a sandwich system whereby an HRP-conjugate streptavidin secondary antibody is used to reveal the reaction. A standard curve was also included as internal control, and samples were tested in duplicate to ensure reproducible results.

\section{Statistical analysis}

Continuous non Gaussian distributed variables were transformed into natural logarithms and described, after back transformation, as means and 95\% confidence intervals. Comparisons between independent groups were performed with Student's t test, and among more than two groups, with a generalized linear model. Multiple comparisons were performed by means of Tukey grouping. Pearson's coefficients were determined to evaluate correlations between continuous variables. To evaluate the prediction of S-SCCA as a function of tissue marker values, a linear regression model was set up with the natural logarithm of S-SCCA as the dependent variable and the natural logarithm of the tumoral and/or peritumoral SCCA value as the independent variable. To evaluate the linear relation of tumoral, peritumoral and serum SCCA as a function of nodule size, a regression model was performed only on single nodule observations.

To evaluate the diagnostic accuracy of serum SCCA a ROC analysis was performed; cut-off value and related sensitivity, specificity, and Youden index were determined.

Qualitative variables were summarized as count and percentage. Comparisons between independent groups were performed with chi-square test or Fisher's exact test when appropriate.

All tests were considered statistically significant at a pvalue of $<0.05$. Analyses were performed with SAS System software for PC, version 9.1.

\section{Abbreviations}

HCC: Hepatocellular carcinoma; LC: liver cirrhosis; SCCA: squamous cellular carcinoma antigen; AFP: alphafetoprotein; -IC: immunocomplex.

\section{Competing interests}

The authors declare that they have no competing interests.

\section{Authors' contributions}

PT performed the statistical analysis. EF, UA, GA, AM and AM performed the experiments. LL supervised the collection of biological samples. SA supervised the project. GG supervised the project and prepared the manuscript. All authors read and approved the manuscript.

\section{Acknowledgements}

This study was supported by the Italian Association Cancer Research (AIRC), (grant to GG number 202240GNN28). 


\section{References}

I. Bruix J, Sherman M, Llovet JM, Beaugrand M, Lencioni R, Burroughs AK, Christensen E, Pagliaro L, Colombo M, Rodes J: Clinical management of hepatocellular carcinoma. Conclusions of the Barcelona-2000 EASL conference. European Association for the Study of the Liver. J Hepatol 200I:42I-430.

2. Farinati F, Marino D, DE Giorgio M, Baldan A, Cantarini M, Cursaro C, Rapaccini G, Del Poggio P, Di Nolfo MA, Benvegnu L, Zoli M, Borzio $F$, Bernardi M, Trevisani F: Diagnostic and prognostic role of alpha-fetoprotein in hepatocellular carcinoma: both or neither? Am J Gastroenterol 2006:524-532.

3. Giannelli G, Antonaci S: New frontiers in biomarkers for hepatocellular carcinoma. Dig Liver Dis 2006:854-859.

4. Marrero JA, Lok AS: Newer markers for hepatocellular carcinoma. Gastroenterology 2004:SII3-SII9.

5. Giannelli G, Fransvea E, Trerotoli P, Beaugrand M, Marinosci F, Lupo L, Nkontchou G, Dentico P, Antonaci S: Clinical validation of combined serological biomarkers for improved hepatocellular carcinoma diagnosis in $96 \mathrm{I}$ patients. Clin Chim Acta 2007:| $47-152$.

6. Suminami Y, Kishi F, Sekiguchi K, Kato H: Squamous cell carcinoma antigen is a new member of the serine protease inhibitors. Biochem Biophys Res Commun 1991:51-58.

7. Pontisso P, Calabrese F, Benvegnu L, Lise M, Belluco C, Ruvoletto MG, De Falco S, Marino M, Valente M, Nitti D, Gatta A, Fassina G: Overexpression of squamous cell carcinoma antigen variants in hepatocellular carcinoma. Br J Cancer 2004:833-837.

8. Giannelli G, Marinosci F, Sgarra C, Lupo L, Dentico P, Antonaci S: Clinical role of tissue and serum levels of SCCA antigen in hepatocellular carcinoma. Int J Cancer 2005:579-583.

9. Guido M, Roskams T, Pontisso P, Fassan M, Thung SN, Giacomelli L, Sergio A, Farinati F, Cillo U, Rugge M: Squamous cell carcinoma antigen in human liver carcinogenesis. J Clin Pathol 2008, 6 I (4):445-7.

10. Pontisso P, Quarta S, Caberlotto C, Beneduce L, Marino M, Bernardinello E, Tono N, Fassina G, Cavalletto L, Gatta A, Chemello L: Progressive increase of SCCA-IgM immune complexes in cirrhotic patients is associated with development of hepatocellular carcinoma. Int J Cancer 2006:735-740.

II. Uemura Y, Pak SC, Luke C, Cataltepe S, Tsu C, Schick C, Kamachi Y, Pomeroy SL, Perlmutter DH, Silverman GA: Circulating serpin tumor markers SCCAI and SCCA2 are not actively secreted but reside in the cytosol of squamous carcinoma cells. Int J Cancer 2000:368-377.

12. Prospective validation of the CLIP score: a new prognostic system for patients with cirrhosis and hepatocellular carcinoma. The Cancer of the Liver Italian Program (CLIP) Investigators. Hepatology 2000:840-845.

13. Giannelli G, Bergamini C, Fransvea E, Marinosci F, Quaranta V, Antonaci S: Human Hepatocellular Carcinoma (HCC) Cells Require Both alpha3betal Integrin and Matrix Metalloproteinases Activity for Migration and Invasion. Lab Invest 2001:6I3-627. 